Cite as: Maaroof, B.B., Rashid, T.A., Abdulla, J.M. et al. (2022). Current Studies and Applications of Shuffled Frog Leaping Algorithm: A Review. Arch Computat Methods Eng. https://doi.org/10.1007/s11831-021-09707-2

\title{
Current Studies and Applications of Shuffled Frog Leaping Algorithm: A Review
}

Bestan B. Maaroof ${ }^{1}$; Tarik A. Rashid²; Jaza M. Abdulla ${ }^{3,4}$; Bryar A. Hassan ${ }^{5}$; Abeer Alsadoon ${ }^{6,7}$; Mokhtar Mohammadi'; Mohammad Khishe9; Seyedali Mirjalilii10,11

${ }^{1}$ Information Technology Department, College of Commerce, University of Sulaimani, KRI, Iraq

${ }^{2}$ Computer Science and Engineering, University of Kurdistan Hewler, Erbil, KRI, Iraq.

${ }^{3}$ Computer Science Department, College of Science, Komar University of Science and Technology,

Sulaymaniyah, KRI, Iraq.

${ }^{4}$ Information Technology, College of Commerce, University of Sulaimani, Sulaymaniyah, KRI, Iraq.

${ }^{5}$ Kurdistan Institution for Strategic Studies and Scientific Research, Sulaimani, Iraq

${ }^{6}$ School of Computing and Mathematics, Charles Sturt University, Sydney, Australia

${ }^{7}$ Asia Pacific International College (APIC), Information Technology Department, Sydney, Australia

${ }^{8}$ Department of Information Technology, College of Engineering and Computer Science, Lebanese French

University, Kurdistan Region, Iraq

${ }^{9}$ Department of Marine Electronics and Communication Engineering, Imam Khomeini Marine Science

University, Nowshahr, Iran

${ }^{10}$ Centre for Artificial Intelligence Research and Optimization, Torrens University, Australia

${ }^{11}$ Yonsei Frontier Lab, Yonsei University, Seoul, Korea

Emails (corresponding): bryar.hassan@kissr.edu.krd; tarik.ahmed@ukh.edu.krd

\begin{abstract}
Shuffled Frog Leaping Algorithm (SFLA) is one of the most widespread algorithms. It was developed by Eusuff and Lansey in 2006. SFLA is a population-based metaheuristic algorithm that combines the benefits of memetics with particle swarm optimization. It has been used in various areas, especially in engineering problems due to its implementation easiness and limited variables. Many improvements have been made to the algorithm to alleviate its drawbacks, whether they were achieved through modifications or hybridizations with other well-known algorithms. This paper reviews the most relevant works on this algorithm. An overview of the SFLA is first conducted, followed by the algorithm's most recent modifications and hybridizations. Next, recent applications of the algorithm are discussed. Then, an operational framework of SLFA and its variants is proposed to analyze their uses on different cohorts of applications. Finally, future improvements to the algorithm are suggested. The main incentive to conduct this survey to provide useful information about the SFLA to researchers interested in working on the algorithm's enhancement or application.
\end{abstract}

\section{Keywords}

Shuffled Leap-Frog Algorithm (SFLA), Hybridization, Modification Application, Review. 
Cite as: Maaroof, B.B., Rashid, T.A., Abdulla, J.M. et al. (2022). Current Studies and Applications of Shuffled Frog Leaping Algorithm: A Review. Arch Computat Methods Eng. https://doi.org/10.1007/s11831-021-09707-2

\section{Introduction}

The SFLA is originated as a result of an investigation by Eusuf and Lansey in 2003. They combined the ideas used in the Shuffled Complex Evolution algorithm (SCE) [1] and Particle Swarm Optimization (PSO) [2]. Consequently, they come up with a new meta-heuristic for tackling discrete and/or combinatorial problems. The basic architecture of the SFLA is based on the assumptions that knowledge is shared socially between the population, providing an evolutionary benefit [3].

SFLA is a methodology, which depends on imitation of the behavior patterns of frogs, taking into account a crowd of frogs leaping in a swamp, on the lookout for the place, which has the highest food quantity reachable, in which the swamp has multiple stones at distinct points that make it easier for the frogs to step on. The aim is to identify a stone with the maximum food amount available. Communicating between frogs can progress their memes as infection can be propagated among them. As a result of improvement in memes, each frog's position will be changed by tuning its leaping step size [4]. The population is meant to be a number of solutions which is represented as a swarm of frogs that is segregated into subgroups denoted to as memeplexes. Each memeplex represented a distinct frog community, each conducting a local quest. The specific frogs grip ideas within each memeplex, which can be persuaded by further frogs' ideas and progress through a memetic evolution process. In a shuffling process, ideas are passed between memeplexes exactly after several memetic evolution phases [5].

This algorithm's main advantages are its fast convergence and accuracy in searching for global solutions [6]. The first SFLA proposal was implemented and used to construct the water distribution network in an optimized way by [3] in 2003. In several various applications, it has been successfully used and gives satisfactory performance [7]. The original algorithm also reveals some drawbacks, such as non-uniform initial population, local and adaptive power limitations, and premature convergence [8].

It is evident from the literature that extensive surveys on certain algorithms inspired by nature, have been performed, such as PSO [9], Whale Optimization Algorithm (WOA) [10], Dragonfly Algorithm(DA) [11], and Backtracking Search optimization Algorithm (BSA) [12, 13]. Nonetheless, no recent survey or research work has been conducted on SFLA since 2014 as it is a very distinguished algorithm and has many applications and publications rely on it to provide a summary on the algorithm, its adjustments, its hybridizations, and its applications. After its initial journal publishing, SFLA has gained widespread recognition. It has subsequently achieved widespread acceptance in a variety of engineering and medical sectors, resulting in an enormous amount of 
published work, as shown in Figure (1). It demonstrates a sustained and increasing interest in the SFLA method and its variants, most notably since 2006 in terms of publications on its use, but also in terms of significant algorithm layout modifications since 2006. We plan to include a thorough overview of SFLA in this analysis in order to provide a valuable resource for researchers looking for inspiration for emerging technology in a variety of realistic applications. We would make every attempt to implement the majority of these developments.

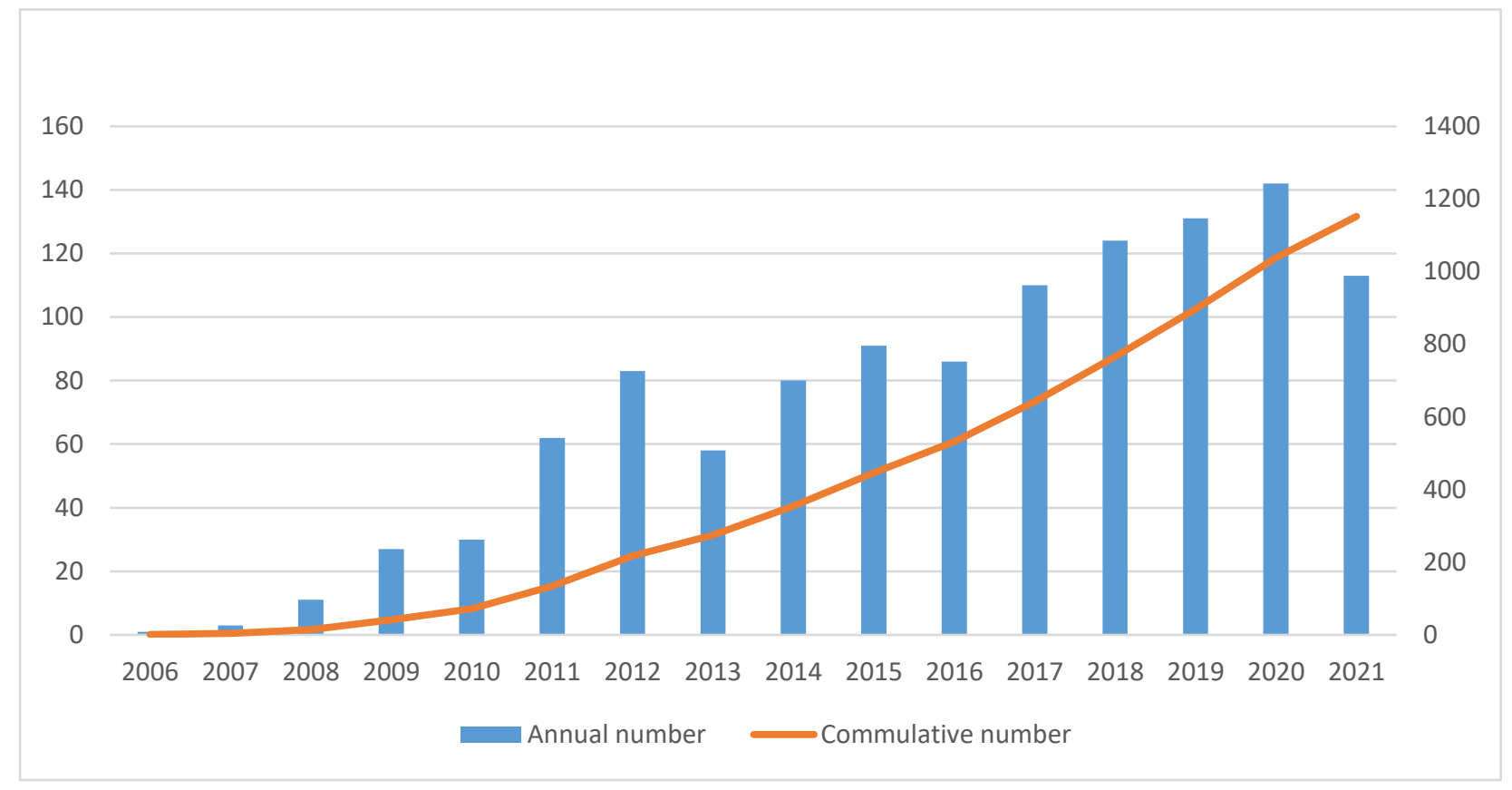

Figure (1): Growing interest in the analysis and application of SFLA since 2006 (Source: Google Scholar, keyword search: "Shuffled Frog Leaping Algorithm", at 10: 42 Wednesday $3^{\text {rd }}$ November 2021)

The main reason behind conducting this review is to help researchers use it in different areas or propose modifications to it and hybridize it with other common algorithms. The purpose of this analysis consisted of several aspects: first, the most research developments and studies carried out on SFLA were highlighted, where hybridization models were used to combine SFLA with other techniques to improve the resulting algorithm's efficiency. Second, this research has concentrated of adjustment that have been applied to SFLA to enhance its power to look for the best solution. Third, much of the research work related to different applications applied to SFLA has been collected. In return, this research work would also open the way for researchers to introduce other improvements to the SFLA algorithm to match their various purposes in diverse fields.

The outline of this paper starts with summarizing the previous literature work on SFLA, followed by describing SFLA with its characteristics in Section 2. Additionally, various SFLA modifications are presented and explained and in Section 3. Section 4 is about the extensions of SFLA. Next, various 
Cite as: Maaroof, B.B., Rashid, T.A., Abdulla, J.M. et al. (2022). Current Studies and Applications of Shuffled Frog Leaping Algorithm: A Review. Arch Computat Methods Eng. https://doi.org/10.1007/s11831-021-09707-2

applications of SFLA are presented in Section 5. Then, an operational framework of SLFA and its variants is proposed in Section 6 to analyze their uses on different cohorts of applications. Finally, after summarizing the enhancements and evaluating future research directions, we conclude the key achievements form this review article.

\section{Related Work}

This section outlines the existing review work on SFLA. This is the only survey on SFLA conducted by [7]. It was about a survey on the role of basic, modified and hybrid SFLA on optimization problems. The article contrasted prior studies on SFLA and its usefulness to the most widely used optimization algorithms. Referencing to the previous literature work, many efforts by previous researchers on SFLA denote the next generations of basic SFLA with diverse structures for modified SFLA or hybrid SFLA. As well, an attempt is made to highlight these structures, their enhancements and advantages. Additionally, this review paper discussed the top SFLA improvements for solving multi-objective optimization issues, including optimizing local and global discovery, preventing local optima, decreasing computational time, and improving the efficiency of the initial community. The calculated improvements in SFLA focused on statistical analysis of 89 reported articles and taking into account the most often used and successful changes made by a significant number of researchers. Finally, the quantitative validations address the SFLA as a versatile algorithm that outperformed other optimization algorithms in a variety of applications.

Our review study distinguishes itself from the earlier survey work in a variety of ways, as detailed below: (1) The previous study is out of date, having been published in 2014, and it did not concentrate on different types of applications, such as engineering, power and energy, and computing. This overview discusses the most current advancements in a variety of applications from 2006 to 2021. (2) The implementation of SFLA is described in detail, including a step-by-step approach and a practical example. (3) We also discuss the state-of-the-art reviews on SFLA and its derivatives. (4) An operational framework for SFLA is presented in order to examine its core implementation and extensions in real-world applications and to establish links between these versions. (5) It not only demonstrates the difficulties of SFLA, but also suggests possible solutions. We identified over 500 recent research papers by entering different versions of the keyword ' shuffled frog-leaping algorithm and SFLA' into the Google Scholar search box. Following a rigorous study of the gathered papers, we picked around 70 as the most significant in terms of SFLA and its variations on practical applications, which are addressed in this study. 
Cite as: Maaroof, B.B., Rashid, T.A., Abdulla, J.M. et al. (2022). Current Studies and Applications of Shuffled Frog Leaping Algorithm: A Review. Arch Computat Methods Eng. https://doi.org/10.1007/s11831-021-09707-2

\section{The standard SFLA}

As a meta-heuristic, the SFLA was suggested, and it focuses on the progression of memes between collaborating individuals and the information global exchange. Conventional mathematical models are based on population principles; thus, a group of agents is a population. The agent has the corresponding utility value, which controls the individual value. Time is separated into time loop steps, which are discreet. Figure (2) illustrates the SFLA flowchart. The algorithm is defined in the following subsections. 
Cite as: Maaroof, B.B., Rashid, T.A., Abdulla, J.M. et al. (2022). Current Studies and Applications of Shuffled Frog Leaping Algorithm: A Review. Arch Computat Methods Eng. https://doi.org/10.1007/s11831-021-09707-2

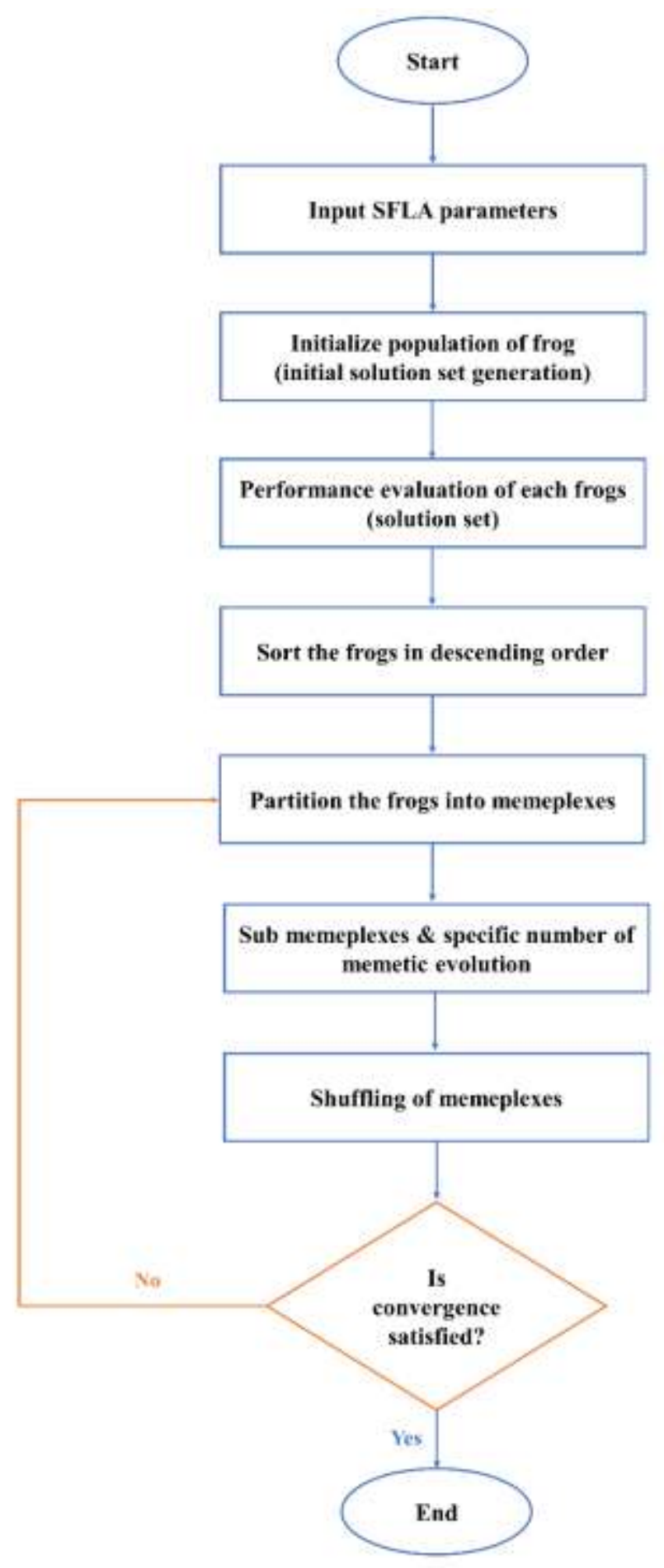

Figure (2): The flowchart of SFLA 
Cite as: Maaroof, B.B., Rashid, T.A., Abdulla, J.M. et al. (2022). Current Studies and Applications of Shuffled Frog Leaping Algorithm: A Review. Arch Computat Methods Eng. https://doi.org/10.1007/s11831-021-09707-2

\subsection{The Standard SFLA}

The population comprises a swarm of frogs or solutions, split up into subgroups identified as memeplexes [14]. Each frog consists of memo types (decision variable parameter). The population of frogs is shown as $U_{1}, U_{2}, \ldots, U_{F}$ and the frog $i$ is presented as a decision vector variable such as $U_{i}=\left\{U_{i}^{1}, U_{i}^{2}, \ldots, U_{i}^{d}\right\}, d$ signifies the number of decision variables in a frog. Memeplexes each conducting a local search. After a certain number of evolution stages, ideas are transferred across memeplexes through a shuffling mechanism. Both the local search and shuffle processes are continued until the convergence criteria stated are satisfied.

In the two phases, the SFLA meta-heuristic technique is outlined [3, 15]:

1) A phase of the global exploration consists of the following steps:

a. Step One: Initialization of Population

i. specify the memeplexes number; $m$.

ii. specify the frog's number in each memeplex; $n$

iii. Generate the population; $F=m \times n$

b. Step Two: Generation of virtual population.

i. Generate sample $F$; virtual frogs $U_{1}, U_{2}, \ldots, U_{F}$ in the achievable space $\Omega \subset ね^{d} . d$ signifies decision variables' number, for instance, memotype (s) number in a meme held by a frog.

ii. Calculate the fitness function for each frog,

c. Step Three: Frogs ranking.

i. arrange the $F$ frogs in descending order based on their fitness function and place them in a list $X ; X=\left\{U_{\mathrm{i}}, f_{(\mathrm{i})}, \mathrm{i}=1, \ldots, \mathrm{F}\right\}$, so as $\mathrm{i}=1$ characterizes the best global frog $\left(P_{x}\right)$, where $P_{x}=U_{1}$.

d. Step Four: Divide frogs into memeplexes. For example, memeplex 1 gets rank 1, memeplex 2 gets rank 2, memeplex $m$ gets rank $m$, memeplex 1 gets rank $m+1$, this process continue until all frogs has been partitioned on the memeplexes.

e. Step Five: Evolution of Memetic inside each memeplex.

i. Entering the phase of local exploration.

f. Step Six: Mix up memeplexes.

g. Step Seven: Verify convergence.

i. Terminate if criteria of convergence are met. If not, go back to step 3. 
2) The phase of Local exploration: Each memeplex progression continues autonomously in Step Four of the global search $N$ times. The algorithm returns to global shuffling exploration after the memeplexes have been progressed. The steps of the local exploration phase are described in detail for each memeplex in the local search.

a. Step One: Set the counters for no. of memeplexes $(m)$ and counting evolutionary steps $\mathrm{N}$ in each memeplex such that:

i. $\quad i m=m+1$

ii. $i N=N+1$

b. Step Two: Form a sub-memeplex for each memeplex.

i. The sub-memeplex suggested technique assigns greater weight to frogs with greater performance and fewer weight frogs to lower performance. Through a triangular probability distribution, the weights are allocated as in Equation (1) [3].

$p_{n}=\frac{2(n+1-j)}{n(n+1)} \quad$ Where $j=1,2, \ldots, n$

Here, a submemeplex of array $Z$ is formed by a random selection of $q$ distinct frogs from $\mathrm{n}$ frogs in each memeplex. The frogs arranged in decreasing order such that $(i q=1)$ is the frog's best position $\left(P_{B}\right)$ and $(i q=q)$ is the frog's worst position $\left(\mathrm{P}_{W}\right)$. The idea of the submemeplex is exemplified in Figure (3).

c. Step Three: Calculate step size S with Equation (2) [3] and enhance the position of the worst frog using Equation (3) [3].

step size $S=\min \left\{\operatorname{int}\left[\operatorname{rand}\left(P_{B}-P_{W}\right)\right], S_{\max }\right\} \quad$ for a positive step

step size $S=\max \left\{\right.$ int $\left[\right.$ rand $\left.\left.\left(P_{B}-P_{W}\right)\right], S_{\max }\right\} \quad$ for a negative step

rand signifies the random number between ranges from zero to one and $\mathrm{S}_{\max }$ signifies the maximum step size allowed by a frog to jump.

$U_{(q)}=P_{W}+S$

Where $U_{(q)}$ shows a new place in the next iteration (See Figure (4)).

d. Step Four: if new $U_{(q)}>$ old $U_{(q)}$ then go to the local exploration step, otherwise, go to Step Five.

e. Step Five: Select the global best frog position $P_{X}$.

i. Find new step size $\mathrm{S}$ by:

Step size $S=\min \left\{\operatorname{int}\left[\operatorname{rand}\left(P_{X}-P_{W}\right)\right], S_{\max }\right\}$

for a positive step

Step size $S=\max \left\{\operatorname{int}\left[\operatorname{rand}\left(P_{X^{-}} P_{W}\right)\right], S_{\max }\right\}$ for a negative step 
ii. Then, calculate the new position through Equation (3).

\section{f. Step Six:}

$i$. If $U_{(q)}$ could not resolve inside viable space, then calculate the new performance, or else, move to Step Six.

ii. If the value of $f_{(\mathrm{q})}$ was better, then switch the old frog with the new one and move to Step Seven, otherwise move to Step Six.

g. Step Seven: Generate a new frog randomly.

h. Step Eight: upgrade the memeplex.

i. Step Nine: Move to Step Two, If $(i N<N)$,

j. $\quad$ Step Ten: Move to Step 1, If $(\mathrm{im}<m)$, otherwise, move back the global search to shuffle memeplexes.

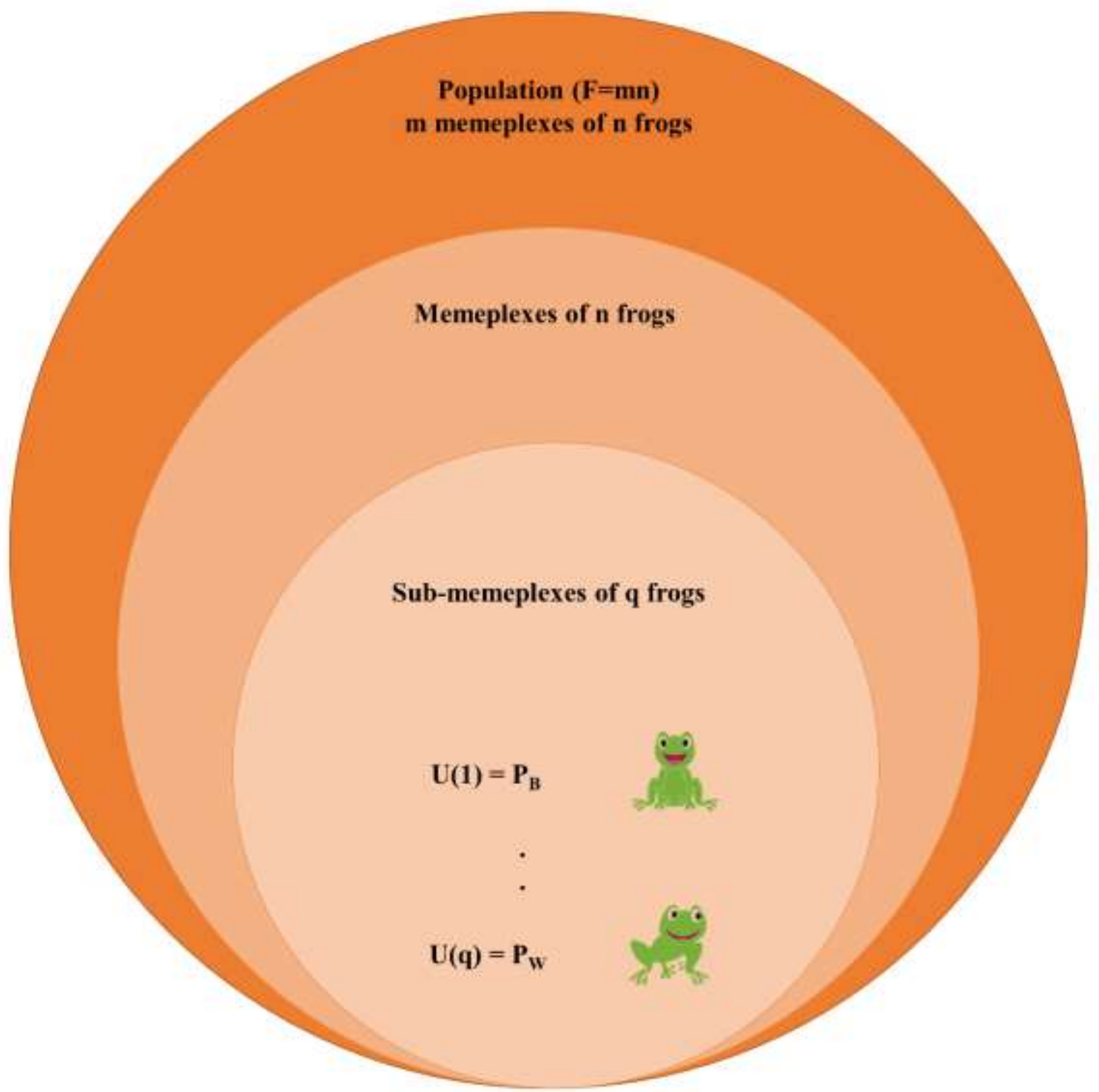

Figure (3): An example of the idea of a sub memeplex. $P_{B}$ correspond to the position $U_{(1)}$ and $P_{W}$ correspond to the position $U_{(q)}$. 
Meme of the worst frog in the sub-memeplex $\left(P_{w}\right)$
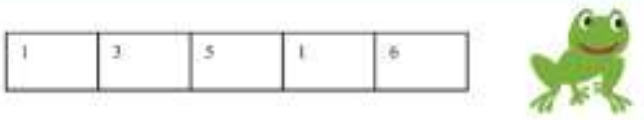

Meme of the best frog in the sub-memeplex $\left(P_{B}\right)$
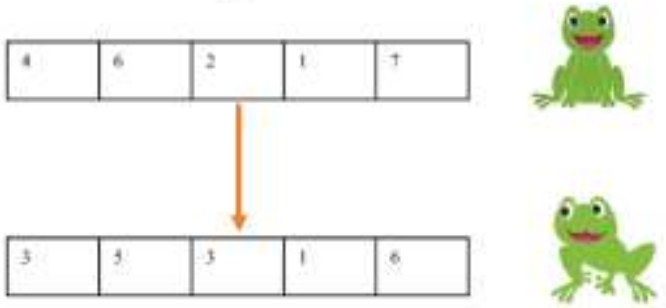

New meme of the worst frog in the sub-memeplex

Figure (4): An illustration of the memetic evolution in a sub-memeplex

\subsection{Hyperparameter Tuning for SFLA}

Like all the other heuristic algorithms, selecting parameters is essential for the results of SFLA. SFLA consists of the following parameters:

1) $m$, signifies memeplexes number.

2) $n$, signifies frogs' number in a memeplex

3) q signifies frogs' number in a sub-memeplex

4) $N$ signifies infection steps number between two consecutive shuffling in a memeplex.

5) $\mathrm{S}_{\max }$ signifies the size of the maximum step permitted in the course of an evolutionary step.

The hyperparameter tuning for SFLA and their impacts on algorithm output are shown in Table (1).

Table (1): Hyperparameter Tuning for SFLA

\begin{tabular}{|c|l|l|l|}
\hline Parameter & Description & $\begin{array}{l}\text { Selection of small no. of the parameter } \\
\text { leads to }\end{array}$ & $\begin{array}{l}\text { Selection of large no. of the } \\
\text { parameter leads to }\end{array}$ \\
\hline $\mathrm{F}=(\mathrm{mn})$ & $\begin{array}{l}\text { The most } \\
\text { significant } \\
\text { parameter is the } \\
\text { number of } \mathrm{F} .\end{array}$ & $\begin{array}{l}\text { Increases locating the global optimum or } \\
\text { near optimum probability. }\end{array}$ & $\begin{array}{l}\text { Making it more computationally } \\
\text { burdensome. }\end{array}$ \\
\hline $\mathrm{m}, \mathrm{n}, \mathrm{q}$ & $\begin{array}{l}\text { It is necessary to } \\
\text { ensure that } \mathrm{n} \text { is } \\
\text { not too small }\end{array}$ & $\begin{array}{l}\text { If too few frogs were presented in each } \\
\text { memeplex, the use of the local memetic } \\
\text { evolution strategy is lost. } \\
\text { The output reaction of the algorithm to } q \text { is } \\
\text { that the exchange of information is slow if } \\
\text { too few frogs are chosen in a submemeplex, } \\
\text { resulting in greater solution times. }\end{array}$ & $\begin{array}{l}\text { When several frogs were picked, the } \\
\text { to extend the searching time. } \\
\text { frogs were contaminated with } \\
\text { undesirable thoughts that cause the }\end{array}$ \\
\hline
\end{tabular}


Cite as: Maaroof, B.B., Rashid, T.A., Abdulla, J.M. et al. (2022). Current Studies and Applications of Shuffled Frog Leaping Algorithm: A Review. Arch Computat Methods Eng. https://doi.org/10.1007/s11831-021-09707-2

\begin{tabular}{|c|l|l|l|}
\hline $\mathrm{N}$ & $\begin{array}{l}\text { Any value } \\
\text { bigger than } 1 \text { can } \\
\text { be taken. }\end{array}$ & $\begin{array}{l}\text { The memeplexes will be shuffled regularly, } \\
\text { decreasing the exchange of ideas on a local } \\
\text { scale. }\end{array}$ & $\begin{array}{l}\text { Each memeplex can shrink into a local } \\
\text { optimum. }\end{array}$ \\
\hline $\mathrm{S}_{\max }$ & $\begin{array}{l}\text { A limitation on } \\
\text { regulating the } \\
\text { global } \\
\text { exploration } \\
\text { capacity of the } \\
\text { SFLA. }\end{array}$ & $\begin{array}{l}\text { It decreases the global exploration, } \\
\text { rendering the algorithm a local search. }\end{array}$ & $\begin{array}{l}\text { The true optimum could be lacking, } \\
\text { since it is not fine-tuned. }\end{array}$ \\
\hline
\end{tabular}

\section{Variants of SFLA}

Due to the SFLA's success, several publications have been suggested, and many of them have been implemented in multiple implementations to improve the efficiency of the original SFLA [3]. Scholars were motivated to improve the performance of standard SFLA in light of its supposed shortcomings in terms of deficiency and convergence speed. Numerous authors have suggested revisions to the initial SFLA, although others have attempted adaptations to address a variety of problems. This segment addresses the evolution of SFLA variants and the present state of knowledge about them. These variations are divided into two groups: SFLA modifications (MSFLA) and SFLA hybridizations (HSFLA). The Figure (5) illustrates a high-level overview of the major SFLA variations.

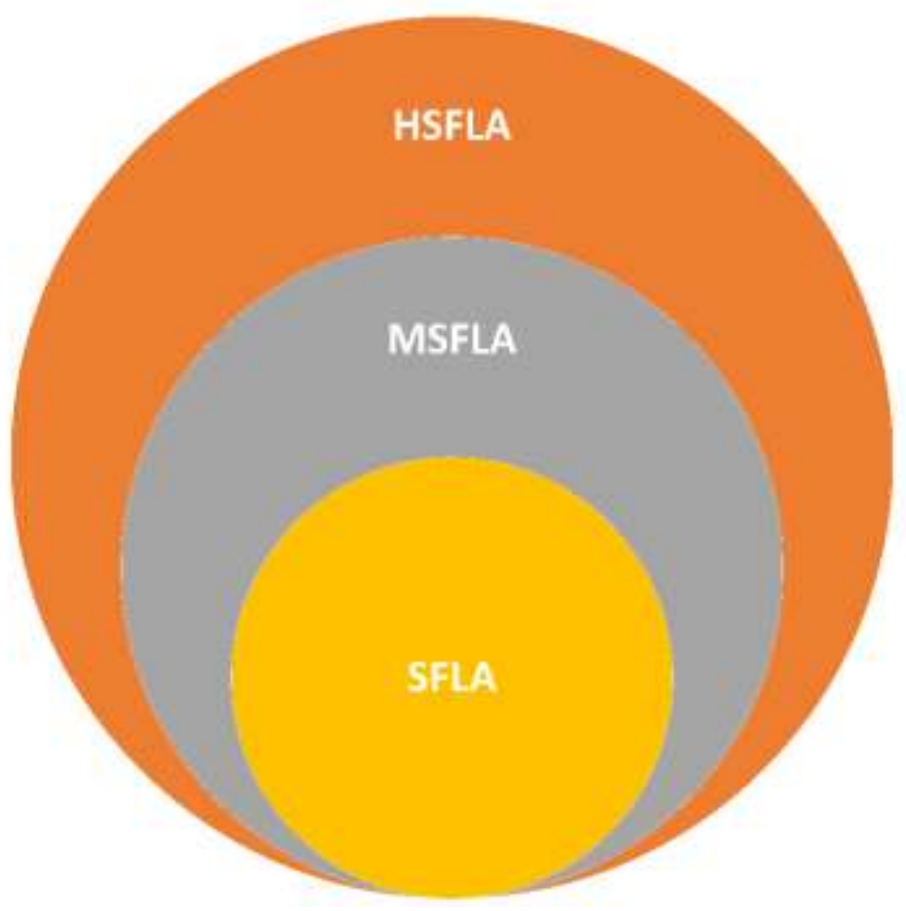

Figure (5): The main variations of SFLA 


\subsection{Modifications}

Scholars have revised SFLA multiple times to improve its effectiveness and efficiency and to make it more fit for a specific use. This section examines nine different SFLA algorithms that have been modified. MSFLA, bilevel SFLA, two-phase SFLA, ISFLA, MCSFLA, MMSFLA, DSFLA, rMQSFLA, and MGSFLA are the modified SFLA. Table (2) summarizes these algorithms. Some researchers in various areas have modified SFLA. Generally, the modifications are made in local search, global search, shuffling, memeplex, and leaping processes.

Table (2): The modified SFLA algorithms

\begin{tabular}{|c|c|c|c|c|c|c|}
\hline Algorithm & $\begin{array}{l}\text { Reference, } \\
\text { author(s) }\end{array}$ & Aim & $\begin{array}{l}\text { Modification } \\
\text { area }\end{array}$ & Methodology & Application & Results \\
\hline \multirow[t]{6}{*}{ MSFLA } & $\begin{array}{l}\text { [16], E. E. } \\
\text { Elattar }\end{array}$ & $\begin{array}{l}\text { Solving } \\
\text { emission and } \\
\text { economic } \\
\text { dispatch } \\
\text { (CHEED) } \\
\text { issue by the } \\
\text { use of green } \\
\text { energy. }\end{array}$ & $\begin{array}{l}\text { Global and local } \\
\text { search processes } \\
\text { in SFLA }\end{array}$ & $\begin{array}{l}\text { Modifying } \\
\text { the global and } \\
\text { local search } \\
\text { processes in } \\
\text { SFLA }\end{array}$ & $\begin{array}{l}\text { CHEED } \\
\text { problem with } \\
\text { renewable } \\
\text { sources }\end{array}$ & $\begin{array}{l}\text { The superiority } \\
\text { of MSFLA over } \\
\text { other methods } \\
\text { was } \\
\text { demonstrated. }\end{array}$ \\
\hline & $\begin{array}{l}{[17], \quad \text { M. }} \\
\text { Moazzami } \\
\text { et al. }\end{array}$ & $\begin{array}{l}\text { Solving the } \\
\text { positioning } \\
\text { and size of } \\
\text { dispersed } \\
\text { generating } \\
\text { units and D- } \\
\text { STATCO }\end{array}$ & $\begin{array}{l}\text { Local search } \\
\text { (Interactive } \\
\text { updating of worst } \\
\text { frog) }\end{array}$ & $\begin{array}{l}\text { 1) Widening } \\
\text { the frog leap } \\
\text { space } \\
\text { 2) Subgroup } \\
\text { definitions }\end{array}$ & $\begin{array}{l}\text { Distributed } \\
\text { generators }\end{array}$ & $\begin{array}{l}\text { MSFLA was } \\
\text { more effective } \\
\text { compared with } \\
\text { the results } \\
\text { achieved from } \\
\text { genetic } \\
\text { algorithm (GA). }\end{array}$ \\
\hline & $\begin{array}{l}\text { [18], M. H. } \\
\text { Oboudi et } \\
\text { al. }\end{array}$ & $\begin{array}{l}\text { Providing a } \\
\text { heuristic } \\
\text { strategy for the } \\
\text { purposeful } \\
\text { islanding of } \\
\text { microgrids }\end{array}$ & $\begin{array}{l}\text { Problem solution } \\
\text { space; bus } \\
\text { voltage; load } \\
\text { controllability; } \\
\text { load priority; the } \\
\text { ability to } \\
\text { construct larger } \\
\text { islands; and line } \\
\text { capacity } \\
\text { constraints; }\end{array}$ & $\begin{array}{l}\text { Employing } \\
\text { sub- } \\
\text { memeplex }\end{array}$ & Microgrids & $\begin{array}{l}\text { MSFLA is the } \\
\text { ideal approach } \\
\text { for TKP } \\
\text { solution }\end{array}$ \\
\hline & $\begin{array}{l}\text { [19], D. } \\
\text { Mora-Melia } \\
\text { et al. }\end{array}$ & $\begin{array}{l}\text { Water } \\
\text { distribution } \\
\text { network }\end{array}$ & $\begin{array}{l}\text { Sub-memeplex } \\
\text { parameter q }\end{array}$ & $\begin{array}{l}\text { Optimization } \\
\text { in parameter } \\
\text { setting }\end{array}$ & $\begin{array}{l}\text { Water } \\
\text { distribution } \\
\text { networks }\end{array}$ & $\begin{array}{l}\text { The efficiency } \\
\text { of MSFLA } \\
\text { significantly } \\
\text { improved. }\end{array}$ \\
\hline & $\begin{array}{l}{[20], \quad \text { A. }} \\
\text { Baziar et al. }\end{array}$ & $\begin{array}{l}\text { Improving } \\
\text { total } \\
\text { searchability } \\
\text { of the method } \\
\text { and avoiding } \\
\text { premature } \\
\text { convergence }\end{array}$ & $\begin{array}{l}\text { Two-phase } \\
\text { modification } \\
\text { (Lévy flight, } \\
\text { population) }\end{array}$ & $\begin{array}{l}\text { 1) Using } \\
\text { random walk } \\
\text { 2) Moving the } \\
\text { average of the } \\
\text { population } \\
\text { toward the } \\
\text { best solution. }\end{array}$ & $\begin{array}{l}\text { Nonlinear } \\
\text { and } \\
\text { large scale } \\
\text { problems }\end{array}$ & $\begin{array}{l}\text { MSFLA } \\
\text { showed } \\
\text { superiority over } \\
\text { the methods in } \\
\text { the area. }\end{array}$ \\
\hline & $\begin{array}{l}{[21], \text { T.-H. }} \\
\text { Huynh }\end{array}$ & $\begin{array}{lr}\text { Increasing the } \\
\text { trajectory and } \\
\text { duration of } \\
\text { each frog }\end{array}$ & Local exploration & $\begin{array}{l}\text { Proposing a } \\
\text { new frog } \\
\text { leaping rule }\end{array}$ & $\begin{array}{l}\text { proportional- } \\
\text { integral- } \\
\text { derivative } \\
\text { (PID) } \\
\text { controller }\end{array}$ & $\begin{array}{l}\text { The } \\
\text { effectiveness of } \\
\text { SFLA improved } \\
\text { and premature } \\
\text { convergence } \\
\text { avoided. }\end{array}$ \\
\hline
\end{tabular}




\begin{tabular}{|c|c|c|c|c|c|c|}
\hline & $\begin{array}{l}{[22], \quad \text { E. }} \\
\text { Elbeltagi et } \\
\text { al. }\end{array}$ & $\begin{array}{l}\text { Overcoming } \\
\text { local optimum } \\
\text { and early } \\
\text { convergence }\end{array}$ & Global search & $\begin{array}{l}\text { Introducing a } \\
\text { new } \\
\text { parameter } \\
\text { called a } \\
\text { search } \\
\text { acceleration } \\
\text { factor }(C)\end{array}$ & $\begin{array}{l}\text { Project } \\
\text { management }\end{array}$ & $\begin{array}{l}\text { The } \\
\text { performance of } \\
\text { SFLA } \\
\text { improved. }\end{array}$ \\
\hline $\begin{array}{l}\text { Bilevel } \\
\text { SFLA }\end{array}$ & $\begin{array}{l}{[23], \quad X .} \\
\text { Duan et al. }\end{array}$ & $\begin{array}{l}\text { Solving bi- } \\
\text { level } \\
\text { programming } \\
\text { model }\end{array}$ & $\begin{array}{l}\text { Combination of } \\
\text { two basic models } \\
\text { of SFLAs }\end{array}$ & $\begin{array}{l}\text { Simulating } \\
\text { the process of } \\
\text { finding food } \\
\text { for the frog } \\
\text { population, } \\
\text { which is } \\
\text { separated into } \\
\text { memeplexes }\end{array}$ & $\begin{array}{l}\text { Emergency } \\
\text { vehicle } \\
\text { redistribution } \\
\text { and } \\
\text { dispatching }\end{array}$ & $\begin{array}{l}\text { Bilevel SFLA } \\
\text { was quick and } \\
\text { efficient in } \\
\text { resolving } \\
\text { bilevel } \\
\text { programming } \\
\text { model. }\end{array}$ \\
\hline $\begin{array}{l}\text { Two-phase } \\
\text { SFLA }\end{array}$ & $\begin{array}{ll}{[24],} & \text { B. } \\
\text { Naruka } & \text { et } \\
\text { al. } & \end{array}$ & $\begin{array}{l}\text { Overcoming } \\
\text { the slow } \\
\text { convergence of } \\
\text { SFLA }\end{array}$ & $\begin{array}{l}\text { Applying the elite } \\
\text { Gaussian learning } \\
\text { strategy in the } \\
\text { global } \\
\text { information } \\
\text { exchange phase } \\
\text { and updating the } \\
\text { frog leaping rule } \\
\text { and adding the } \\
\text { learning attitude } \\
\text { of the frogs }\end{array}$ & $\begin{array}{l}\text { 1) } \\
\text { Initialization } \\
\text { of frogs using } \\
\text { evenly } \\
\text { random } \\
\text { generated } \\
\text { integers and } \\
\text { opposition } \\
\text { based } \\
\text { learning } \\
\text { (OBL) } \\
\text { 2) The use of } \\
\text { a scaling } \\
\text { factor for new } \\
\text { frog positions }\end{array}$ & $\begin{array}{l}\text { Structural } \\
\text { design } \\
\text { problems }\end{array}$ & $\begin{array}{l}\text { Increment in } \\
\text { search range } \\
\text { and } \\
\text { enhancement in } \\
\text { the population } \\
\text { diversity were } \\
\text { achieved. }\end{array}$ \\
\hline \multirow[t]{3}{*}{ ISFLA } & $\begin{array}{l}{[25], \quad Z .} \\
\text { Zhen et al. }\end{array}$ & $\begin{array}{l}\text { Extending } \\
\text { discrete } \\
\text { optimization } \\
\text { problem }\end{array}$ & $\begin{array}{l}\text { Distributing the } \\
\text { frogs } \\
\text { memeplexes }\end{array}$ & $\begin{array}{l}\text { Combining } \\
\text { local and } \\
\text { global } \\
\text { searches to } \\
\text { enhances frog } \\
\text { distribution } \\
\text { randomness } \\
\text { and variety in } \\
\text { continuous } \\
\text { space }\end{array}$ & $\begin{array}{l}\text { Continuous } \\
\text { optimization } \\
\text { problem }\end{array}$ & $\begin{array}{l}\text { ISFLA was } \\
\text { capable of } \\
\text { overcoming the } \\
\text { premature } \\
\text { convergence } \\
\text { and sluggish } \\
\text { convergence } \\
\text { speed issues, as } \\
\text { well as } \\
\text { achieving high } \\
\text { optimization } \\
\text { accuracy. }\end{array}$ \\
\hline & $\begin{array}{l}{[26], \quad J .} \\
\text { Siahbalaee } \\
\text { et al. }\end{array}$ & $\begin{array}{l}\text { Relocating and } \\
\text { reconfiguring } \\
\text { distributed } \\
\text { generators }\end{array}$ & $\begin{array}{l}\text { Repositing the } \\
\text { frogs towards a } \\
\text { better position }\end{array}$ & $\begin{array}{l}\text { Repositioning } \\
\text { the frogs }\end{array}$ & $\begin{array}{l}\text { Distributed } \\
\text { generators }\end{array}$ & $\begin{array}{l}\text { The proposed } \\
\text { method had an } \\
\text { advantage over } \\
\text { previous } \\
\text { experiments. }\end{array}$ \\
\hline & $\begin{array}{l}\text { [27], H.-P. } \\
\text { Hsu and T.- } \\
\text { L. Chiang }\end{array}$ & $\begin{array}{l}\text { Examining } \\
\text { complex and } \\
\text { continuous } \\
\text { berth } \\
\text { allocation } \\
\text { problem } \\
\text { (DCBAP) }\end{array}$ & $\begin{array}{l}\text { ISFLA has four } \\
\text { features: } \\
\text { numerous frog } \\
\text { groups, shuffling } \\
\text { method, discrete } \\
\text { operators with } \\
\text { self-adaptive } \\
\text { leap, and self- } \\
\text { adaptive mutation } \\
\text { process }\end{array}$ & $\begin{array}{l}\text { ISFLA } \\
\text { possesses the } \\
\text { following } \\
\text { features: frog } \\
\text { groups, } \\
\text { shuffling } \\
\text { method, } \\
\text { discrete } \\
\text { operators } \\
\text { with self- } \\
\text { adaptive } \\
\text { jump, and } \\
\text { self-adaptive }\end{array}$ & $\begin{array}{l}\text { Berth } \\
\text { allocation } \\
\text { problem }\end{array}$ & $\begin{array}{l}\text { ISFLA } \\
\text { capable } \\
\text { manage } \\
\begin{array}{l}\text { DCBAP at } \\
\text { container } \\
\text { terminal. }\end{array} \\
\end{array}$ \\
\hline
\end{tabular}




\begin{tabular}{|c|c|c|c|c|c|c|}
\hline & & & & $\begin{array}{l}\text { mutation } \\
\text { mechanism }\end{array}$ & & \\
\hline & $\begin{array}{l}{[28], \quad \mathrm{T} .} \\
\text { Zhang et al. }\end{array}$ & $\begin{array}{l}\text { Local } \\
\text { backlight } \\
\text { dimming }\end{array}$ & $\begin{array}{lr}\text { Initial solution } \\
\text { generation and } \\
\text { interval search } \\
\text { solutions }\end{array}$ & $\begin{array}{l}\text { Introducing } \\
\text { cycle } \\
\text { optimization } \\
\text { in ISFLA }\end{array}$ & $\begin{array}{l}\text { Local } \\
\text { dimming } \\
\text { technology }\end{array}$ & $\begin{array}{l}\text { ISFLA delivers } \\
\text { a better trade- } \\
\text { off between } \\
\text { power usage } \\
\text { and picture } \\
\text { quality than } \\
\text { previous image } \\
\text { parameter- } \\
\text { based } \\
\text { algorithms. }\end{array}$ \\
\hline & $\begin{array}{l}{[29], \mathrm{B} . \mathrm{Hu}} \\
\text { et al. }\end{array}$ & $\begin{array}{l}\text { Optimizing } \\
\text { biomedical } \\
\text { data }\end{array}$ & $\begin{array}{l}\text { Chaos memory } \\
\text { weight factor }\end{array}$ & $\begin{array}{l}\text { Introducing } \\
\text { an absolute } \\
\text { balance group } \\
\text { strategy, a } \\
\text { chaos } \\
\text { memory } \\
\text { weight factor, } \\
\text { and an } \\
\text { adaptive } \\
\text { transfer factor }\end{array}$ & $\begin{array}{l}\text { Biomedical } \\
\text { data }\end{array}$ & $\begin{array}{l}\text { ISFLA } \\
\text { improved both } \\
\text { the detection of } \\
\text { related subsets } \\
\text { and } \\
\text { classification } \\
\text { accuracy. }\end{array}$ \\
\hline MCSFLA & $\begin{array}{l}{[30], \mathrm{H} . \mathrm{Pu}} \\
\text { et al. }\end{array}$ & $\begin{array}{l}\text { Optimizing } \\
\text { unmanned } \\
\text { aerial vehicle } \\
\text { (UAV) }\end{array}$ & $\begin{array}{l}\text { Mixing the } \\
\text { concept of GA } \\
\text { PSO }\end{array}$ & $\begin{array}{l}\text { SFLA } \\
\text { combination } \\
\text { with genetic } \\
\text { and social } \\
\text { behavior- } \\
\text { based particle } \\
\text { swarm } \\
\text { optimization }\end{array}$ & $\begin{array}{l}\text { UAV flight } \\
\text { controller }\end{array}$ & $\begin{array}{l}\text { The dynamic } \\
\text { reaction time of } \\
\text { SFLA was very } \\
\text { fast. }\end{array}$ \\
\hline MMSFLA & $\begin{array}{l}{[31], \quad \mathrm{R} .} \\
\text { Azizipanah- } \\
\text { Abarghooee } \\
\text { et al. }\end{array}$ & $\begin{array}{l}\text { Optimizing } \\
\text { multi- } \\
\text { objective } \\
\text { power flow } \\
\text { with flexible } \\
\text { AC } \\
\text { transmission } \\
\text { systems } \\
\text { (FACTS) }\end{array}$ & $\begin{array}{l}\text { Mutation } \\
\text { procedure }\end{array}$ & $\begin{array}{l}\text { A new } \\
\text { mutation with } \\
\text { SFLA }\end{array}$ & $\begin{array}{l}\text { Power flow } \\
\text { with FACTS } \\
\text { devices }\end{array}$ & $\begin{array}{l}\text { MMSFLA was } \\
\text { more effective } \\
\text { compared to } \\
\text { other } \\
\text { algorithms. }\end{array}$ \\
\hline DSFLA & $\begin{array}{l}{[32], \text { J. Cai }} \\
\text { et al. }\end{array}$ & $\begin{array}{l}\text { Minimizing } \\
\text { makespan in } \\
\text { distributed } \\
\text { scheduling } \\
\text { problems } \\
\end{array}$ & $\begin{array}{l}\text { Modification in } \\
\text { local search }\end{array}$ & $\begin{array}{l}\text { Applying } \\
\text { Dynamic } \\
\text { search in each } \\
\text { memeplex }\end{array}$ & $\begin{array}{l}\text { Flow shop } \\
\text { scheduling }\end{array}$ & $\begin{array}{l}\text { It showed } \\
\text { superiority over } \\
\text { the methods in } \\
\text { the area. }\end{array}$ \\
\hline r-MQSFLA & $\begin{array}{l}{[33], \quad Y .} \\
\text { Guo et al. }\end{array}$ & $\begin{array}{l}\text { Solving an } \\
\text { optimization } \\
\text { problem of } \\
\text { many- } \\
\text { objective } \\
\text { allocation of } \\
\text { water } \\
\text { resources for } \\
\text { Inter-Basin } \\
\text { water transfer } \\
\text { IBWT }\end{array}$ & Global Search & $\begin{array}{l}\text { A real-coded } \\
\text { quantum } \\
\text { computer and } \\
\text { an external } \\
\text { dynamic } \\
\text { archive with } \\
\text { SFLA }\end{array}$ & $\begin{array}{l}\text { Water } \\
\text { resources } \\
\text { allocation }\end{array}$ & $\begin{array}{l}\text { r-MQSFLA } \\
\text { performed } \\
\text { admirably in } \\
\text { Jiangsu, China's } \\
\text { Eastern Route } \\
\text { of the South-to- } \\
\text { North Water } \\
\text { Transfer } \\
\text { Project. }\end{array}$ \\
\hline MGSFLA & $\begin{array}{l}\text { [14], D. Lei } \\
\text { and } \mathrm{T} \text {. } \\
\text { Wang }\end{array}$ & $\begin{array}{l}\text { Solving } \\
\text { distributed } \\
\text { two-stage } \\
\text { hybrid flow } \\
\text { shop } \\
\text { scheduling }\end{array}$ & Memeplex & $\begin{array}{l}\text { SFLA with } \\
\text { memeplex } \\
\text { grouping }\end{array}$ & $\begin{array}{l}\text { Flow shop } \\
\text { scheduling }\end{array}$ & $\begin{array}{l}\text { MGSFLA has } \\
\text { most promising } \\
\text { advantage over } \\
\text { the other } \\
\text { methods. }\end{array}$ \\
\hline
\end{tabular}




\subsection{Hybridizations}

Hybridized SFLA is a term that refers to the combination of SFLA with another method. The purpose of hybridized SFLA is to use the strengths of both SFLA and the other algorithms while compensating for their deficiencies. This section discusses eight hybridized BSA algorithms, which are DESFLA, FSA-SFLA, Quantum SFLA, K-means SFLA, SFLA-SVM, SFLA-BFO, and PSFLA. Table (3) summarizes these hybridized algorithms of SFLA.

Table (3): The hybridized SFLA algorithms

\begin{tabular}{|c|c|c|c|c|c|c|}
\hline $\begin{array}{l}\text { Proposed } \\
\text { algorithm }\end{array}$ & $\begin{array}{l}\text { Reference, } \\
\text { author(s) }\end{array}$ & Aim & $\begin{array}{l}\text { Hybridized } \\
\text { with }\end{array}$ & Methodology & Application & Results \\
\hline \multirow[t]{2}{*}{ DESFLA } & $\begin{array}{l}{[34], \quad \text { L. }} \\
\text { Yan et al. }\end{array}$ & $\begin{array}{lr}\text { Tackling } & \text { low } \\
\text { accuracy } & \text { and } \\
\text { avoid } & \text { local } \\
\text { optimum } & \end{array}$ & $\mathrm{DE}$ & $\begin{array}{l}\text { Updating the } \\
\text { strategy of } \\
\text { DE for global } \\
\text { searching } \\
\text { ability }\end{array}$ & $\begin{array}{l}\text { Optimum } \\
\text { power } \\
\text { management } \\
\text { unit (PMU) }\end{array}$ & $\begin{array}{l}\text { The reduction of } \\
\text { the number of } \\
\text { PMU } \\
\text { configuration } \\
\text { was achieved } \\
\text { compared to } \\
\text { DEPSO. }\end{array}$ \\
\hline & $\begin{array}{ll}{[35],} & \text { B. } \\
\text { Naruka } & \text { et } \\
\text { al. } & \end{array}$ & $\begin{array}{l}\text { Improving } \\
\text { searching } \\
\text { capability }\end{array}$ & $\mathrm{DE}$ & $\begin{array}{l}\text { Improving } \\
\text { the searching } \\
\text { capability of } \\
\text { SFLA and } \\
\text { maintaining } \\
\text { the } \\
\text { convergence } \\
\text { of the } \\
\text { population of } \\
\text { frogs }\end{array}$ & $\begin{array}{l}\text { Three } \\
\text { different } \\
\text { types of } \\
\text { chemical } \\
\text { engineering } \\
\text { problems } \\
\text { (linear and } \\
\text { nonlinear) }\end{array}$ & $\begin{array}{l}\text { DESFLA } \\
\text { showed to be } \\
\text { very efficient in } \\
\text { solving } \\
\text { computational } \\
\text { optimization } \\
\text { problems. }\end{array}$ \\
\hline FSA-SFLA & $\begin{array}{l}{[36], \mathrm{Z} . \mathrm{Lu}} \\
\text { et al. }\end{array}$ & $\begin{array}{lr}\text { Finding } & \text { optimal } \\
\text { solution } & \text { region } \\
\text { quickly } & \end{array}$ & $\begin{array}{l}\text { Fish swarm } \\
\text { algorithm } \\
\text { (FSA) }\end{array}$ & $\begin{array}{l}\text { Using the } \\
\text { FSA's ability } \\
\text { for global } \\
\text { optimization, } \\
\text { and SFLA's } \\
\text { ability for } \\
\text { local } \\
\text { optimization }\end{array}$ & Data mining & $\begin{array}{l}\text { FSA-SFLA was } \\
\text { capable of } \\
\text { successfully } \\
\text { reducing } \\
\text { attribute } \\
\text { dimensions } \\
\text { while } \\
\text { maintaining } \\
\text { classification } \\
\text { capability. }\end{array}$ \\
\hline \multirow[t]{2}{*}{$\begin{array}{l}\text { Quantum } \\
\text { SFLA }\end{array}$} & $\begin{array}{l}\text { [37], L. } \\
\text { Wang and } \\
\text { Y. Gong }\end{array}$ & $\begin{array}{l}\text { Achieving a } \\
\text { balance between } \\
\text { global and local } \\
\text { search and to } \\
\text { increase its speed }\end{array}$ & $\begin{array}{l}\text { Quantum } \\
\text { evolutionary } \\
\text { algorithm } \\
\text { (QEA) }\end{array}$ & $\begin{array}{l}\text { Enhancing } \\
\text { the } \\
\text { population } \\
\text { division, } \\
\text { speeding up } \\
\text { convergence, } \\
\text { and } \\
\text { advancing the } \\
\text { success ratio } \\
\text { that reaches } \\
\text { the optimum }\end{array}$ & $\begin{array}{l}\text { 0-1 knapsack } \\
\text { problems }\end{array}$ & $\begin{array}{l}\text { QBSFLA } \\
\text { offered a } \\
\text { number } \\
\text { benefits, } \\
\text { including rapid } \\
\text { convergence, a } \\
\text { strong global } \\
\text { search } \\
\text { capability, and } \\
\text { high stability. }\end{array}$ \\
\hline & $\begin{array}{l}{[38], \text { W. Li }} \\
\text { et al. }\end{array}$ & $\begin{array}{l}\text { Collaborative } \\
\text { recommendation } \\
\text { system }\end{array}$ & $\begin{array}{l}\text { Quantum } \\
\text { computing }\end{array}$ & $\begin{array}{l}\text { Introducing } \\
\text { quantum } \\
\text { computing } \\
\text { into SFLA, } \\
\text { and using } \\
\text { quantum } \\
\text { movement in }\end{array}$ & $\begin{array}{l}\text { Collaborative } \\
\text { filtering }(\mathrm{CF})\end{array}$ & $\begin{array}{l}\text { QBSFLA } \\
\text { enhanced the } \\
\text { predictive } \\
\text { accuracy of } \\
\text { rating scores. }\end{array}$ \\
\hline
\end{tabular}




\begin{tabular}{|c|c|c|c|c|c|c|}
\hline & & & & $\begin{array}{l}\text { the DQSFLA } \\
\text { to search the } \\
\text { local optima }\end{array}$ & & \\
\hline $\begin{array}{l}\text { K-means } \\
\text { SFLA }\end{array}$ & $\begin{array}{l}{[39], \quad \text { Z. }} \\
\text { Tang and } \\
\text { K. Luo }\end{array}$ & $\begin{array}{l}\text { Enhancing } \\
\text { clustering results }\end{array}$ & K-means & $\begin{array}{l}\text { Employing } \\
\text { chaotic local } \\
\text { search, and } \\
\text { introducing a } \\
\text { searching } \\
\text { mechanism } \\
\text { for updating } \\
\text { frog's } \\
\text { position }\end{array}$ & $\begin{array}{l}\text { UCI datasets } \\
\text { (Iris, Wine, } \\
\text { Balance- } \\
\text { scale, and } \\
\text { Housing) }\end{array}$ & $\begin{array}{l}\text { K-means had } \\
\text { better accuracy. }\end{array}$ \\
\hline $\begin{array}{l}\text { SFLA- } \\
\text { SVM }\end{array}$ & $\begin{array}{l}{[9], \text { W. Li }} \\
\text { et al. }\end{array}$ & $\begin{array}{l}\text { Forecasting wind } \\
\text { photovoltaic } \\
\text { battery power }\end{array}$ & SVM & $\begin{array}{l}\text { Burdening } \\
\text { blindness and } \\
\text { randomness } \\
\text { in parameter } \\
\text { selection of } \\
\text { SVM }\end{array}$ & $\begin{array}{l}\text { Wind- } \\
\text { photovoltaic- } \\
\text { battery } \\
\text { generation } \\
\text { system }\end{array}$ & $\begin{array}{l}\text { SFLA-SVM } \\
\text { had higher } \\
\text { optimization } \\
\text { capability. }\end{array}$ \\
\hline $\begin{array}{l}\text { SFLA- } \\
\text { BFO }\end{array}$ & $\begin{array}{l}{[40], Y . \mathrm{Li}} \\
\text { and } \mathrm{Z} . \text { Yan }\end{array}$ & $\begin{array}{l}\text { Reliability } \\
\text { analysis }\end{array}$ & $\mathrm{BFO}$ & $\begin{array}{l}\text { - Using a } \\
\text { strategy of } \\
\text { random } \\
\text { grouping to } \\
\text { maintaining } \\
\text { the diversity } \\
\text { of the } \\
\text { population } \\
\text { - Using the } \\
\text { update } \\
\text { strategy of } \\
\text { Levy flight to } \\
\text { increase the } \\
\text { global search } \\
\text { ability } \\
\text { - Using the } \\
\text { migration } \\
\text { operation } \\
\text { approach to } \\
\text { escape the } \\
\text { local } \\
\text { optimums }\end{array}$ & $\begin{array}{l}\text { System } \\
\text { reliability } \\
\text { model }\end{array}$ & $\begin{array}{l}\text { It was capable } \\
\text { of obtaining the } \\
\text { optimal solution } \\
\text { while } \\
\text { maximizing the } \\
\text { system's } \\
\text { dependability. }\end{array}$ \\
\hline PSFLA & $\begin{array}{l}{[41], \quad \mathrm{X} .} \\
\text { Nie and } \mathrm{H} . \\
\text { Nie }\end{array}$ & $\begin{array}{l}\text { Avoid falling into } \\
\text { the partial } \\
\text { optimal solution, } \\
\text { and to improve } \\
\text { the convergence } \\
\text { speed }\end{array}$ & PSO & $\begin{array}{l}\text { Proposing a } \\
\text { new MPPT } \\
\text { strategy of the } \\
\text { PSFLA } \\
\text { combined } \\
\text { with } \\
\text { recursive } \\
\text { least square } \\
\text { filtering }\end{array}$ & $\begin{array}{l}\text { Maximum } \\
\text { power point } \\
\text { tracking } \\
\text { (MPPT) }\end{array}$ & $\begin{array}{l}\text { It was capable } \\
\text { of successfully } \\
\text { suppressing } \\
\text { measurement } \\
\text { noise effects. }\end{array}$ \\
\hline SFLA-GA & $\begin{array}{l}\text { [42], } \\
\text { Ibrahim GJ } \\
\text { et al. }\end{array}$ & $\begin{array}{l}\text { Optimizing } \\
\text { mobile cloud } \\
\text { service } \\
\text { composition }\end{array}$ & GA & $\begin{array}{l}\text { Embedding } \\
\text { GA with } \\
\text { SFLA to } \\
\text { encode the } \\
\text { individuals } \\
\text { for discrete } \\
\text { problems }\end{array}$ & $\begin{array}{l}\text { Mobile cloud } \\
\text { components }\end{array}$ & $\begin{array}{l}\text { It was achieved } \\
\text { the } \\
\text { improvement in } \\
\text { the } \\
\text { practicability of } \\
\text { the service with } \\
\text { bare minimum } \\
\text { power } \\
\text { utilization, } \\
\text { response time, } \\
\text { and cost for } \\
\text { mobile cloud }\end{array}$ \\
\hline
\end{tabular}




\begin{tabular}{|l|l|l|l|l|l|}
\hline & & & & & $\begin{array}{l}\text { components } \\
\text { according to } \\
\text { some other } \\
\text { algorithms. }\end{array}$ \\
\hline
\end{tabular}

\section{Key Applications of SFLA}

Many publications have been implemented SFLA for real-life applications because of its simplicity in implementation and fast convergence. The key uses of SFLA are in the following domains: water resources management, computing, power generation and energy management, and engineering.

\subsection{Environments and Water Resources}

One of the areas that SFLA used is water resources management. Table (4) briefs the main applications of SFLA in water resources management.

Table (4): Summary of SFLA applications in water resources management

\begin{tabular}{|l|l|l|l|}
\hline Reference, author(s) & Year & Algorithm & Purpose \\
\hline [33], Y. Guo et al. & 2020 & MSFLA & Inter-basin water transfers \\
\hline [43], G. Liu et al. & 2019 & MSFLA & $\begin{array}{l}\text { Solving the optimization problem of } \\
\text { Raman Fiber Amplifier (RFA)of } \\
\text { multi-pumped design }\end{array}$ \\
\hline $\begin{array}{l}\text { [44], T. K. Sharma and D. } \\
\text { Prakash }\end{array}$ & 2019 & SFLA & $\begin{array}{l}\text { Optimization of air pollution } \\
\text { emission }\end{array}$ \\
\hline [45], D. Prakash et al. & 2017 & SFLA & Air pollution control \\
\hline [19], D. Mora-Melia et al. & 2016 & MSFLA & $\begin{array}{l}\text { Water distribution network } \\
\text { optimization problems }\end{array}$ \\
\hline
\end{tabular}

\subsection{Computing}

Another uses of SFLA is computing. These applications are summarized in Table (5).

Table (5): Summary of SFLA applications in computing

\begin{tabular}{|l|l|l|l|}
\hline Reference, author(s) & Year & Algorithm cohort & Purpose \\
\hline [46], B. Boroumand et al. & 2021 & MSFLA & $\begin{array}{l}\text { The mapping process for Network } \\
\text { on Chip (NOC). }\end{array}$ \\
\hline $\begin{array}{l}\text { [47], M. } \\
\text { Mohammadhosseini et al. }\end{array}$ & 2020 & SFLA & $\begin{array}{l}\text { Finding the proper route for the } \\
\text { sensor to send information to the } \\
\text { sink or coordinator. }\end{array}$ \\
\hline [48], R. K. Khadanga et al. & 2020 & HSFLA & $\begin{array}{l}\text { PID controller for affective load } \\
\text { frequency control. }\end{array}$ \\
\hline
\end{tabular}


Cite as: Maaroof, B.B., Rashid, T.A., Abdulla, J.M. et al. (2022). Current Studies and Applications of Shuffled Frog Leaping Algorithm: A Review. Arch Computat Methods Eng. https://doi.org/10.1007/s11831-021-09707-2

\begin{tabular}{|c|c|c|c|}
\hline [49], M. Karpagam et al. & 2020 & MSFLA & \multirow[t]{4}{*}{ Scheduling } \\
\hline [26], Z. Zhen et al. & 2020 & MSFLA & \\
\hline $\begin{array}{l}\text { [50], S. Kayalvili and M. } \\
\text { Selvam }\end{array}$ & 2019 & HSFLA & \\
\hline [51], D. Lei and X. Guo & 2016 & SFLA & \\
\hline [52], M. Karakoyun & 2019 & HSFLA & \multirow[t]{2}{*}{ Traveling salesman problem (TSP). } \\
\hline [8], X. Luo et al. & 2008 & SFLA & \\
\hline [53], L. Wang et al. & 2019 & HSFLA & $\begin{array}{l}\text { Solving the service discovery and } \\
\text { combinatorial optimization of } \\
\text { outsourcing resources (COOR) } \\
\text { problem }\end{array}$ \\
\hline [54], M.-L. Pérez-Delgado & 2019 & SFLA & Quantizing color image. \\
\hline $\begin{array}{l}\text { [55], N. Yuvaraj and A. } \\
\text { Sabari }\end{array}$ & 2017 & SFLA & $\begin{array}{l}\text { Feature selection technique for } \\
\text { Tweeter Sentiment classification. }\end{array}$ \\
\hline [29], B. Hu et al. & 2016 & MSFLA & $\begin{array}{l}\text { Optimizing high-dimensional } \\
\text { biomedical data by feature selection }\end{array}$ \\
\hline
\end{tabular}

\subsection{Power and Energy}

SFLA was mostly used in power generation and energy management. Table (6) presents the key applications of SLFA used in energy and power generation.

Table (6): Summary of SFLA applications in power generation and energy management

\begin{tabular}{|l|l|l|l|}
\hline Reference, author(s) & Year & Algorithmic cohort & Purpose \\
\hline [56], A. Amudha & 2014 & SFLA & $\begin{array}{l}\text { Positioning of Unified } \\
\text { Power Flow Controller in } \\
\text { power line transmitter. }\end{array}$ \\
\hline $\begin{array}{l}\text { [57], M. E. Mosayebian et } \\
\text { al. }\end{array}$ & 2016 & SFLA & $\begin{array}{l}\text { Managing } \\
\text { operational micro-grid. }\end{array}$ \\
\hline [18], M. H. Oboudi et al. & 2016 & MSFLA & $\begin{array}{l}\text { Intentional islanding } \\
\text { control of microgrids. }\end{array}$ \\
\hline [41], X. Nie and H. Nie & 2017 & MSFLA & $\begin{array}{l}\text { Control strategy of } \\
\text { maximum power point } \\
\text { tracking (MPPT) of PV. }\end{array}$ \\
\hline [40], Y. Li and Z. Yan & 2018 & & System reliability analysis. \\
\hline [58], M. Gitizadeh et al. & 2013 & MSFLA & Distribution of generators \\
\hline [14], D. Lei and T. Wang & 2019 & HSFLA & Flow-shop scheduling \\
\hline [26], J. Siahbalaee et al. & 2019 & MSFLA & $\begin{array}{l}\text { Power network } \\
\text { distributions }\end{array}$ \\
\hline
\end{tabular}


Cite as: Maaroof, B.B., Rashid, T.A., Abdulla, J.M. et al. (2022). Current Studies and Applications of Shuffled Frog Leaping Algorithm: A Review. Arch Computat Methods Eng. https://doi.org/10.1007/s11831-021-09707-2

\begin{tabular}{|l|l|l|l|}
\hline [17], M. Moazzami et al. & 2017 & MSFLA & $\begin{array}{l}\text { Sizing and locating } \\
\text { distributed generation } \\
\text { sources. }\end{array}$ \\
\hline [16], E. E. Elattar & 2019 & MSFLA & $\begin{array}{l}\text { Solving the combined heat, } \\
\text { emission, and economic } \\
\text { dispatch (CHEED) with } \\
\text { wind and solar power } \\
\text { problem }\end{array}$ \\
\hline [59], A. S. Reddy and K. & 2013 & HSFLA & $\begin{array}{l}\text { Economic load dispatch } \\
\text { optimization problem }\end{array}$ \\
\hline [60], R. Sridhar et al. & 2017 & SFLA & $\begin{array}{l}\text { Maximum power point } \\
\text { tracking (MPPT). }\end{array}$ \\
\hline [61], S. Nagaraju et al. & 2019 & HSFLA & $\begin{array}{l}\text { Combined Heat and Power } \\
\text { Economic Dispatch } \\
\text { problem (CHPED) }\end{array}$ \\
\hline [62], P. Hu et al. & 2019 & HSFLA & $\begin{array}{l}\text { Optimization of low impact } \\
\text { development (LID) }\end{array}$ \\
\hline [63], H. Chamandoust et al. & 2020 & SFLA & $\begin{array}{l}\text { Tri-objective optimal } \\
\text { performance of a smart } \\
\text { hybrid energy system } \\
\text { (SHES) }\end{array}$ \\
\hline
\end{tabular}

\subsection{Engineering Problems}

SLFA used in several engineering areas. Table (7) lists the use of SFLA in different engineering domains.

Table (7): Summary of SFLA applications in engineering

\begin{tabular}{|l|l|l|l|l|}
\hline Reference, author(s) & Year & Algorithmic cohort & Purpose & Area \\
\hline [23], X. Duan et al. & 2015 & MSFLA & Dispatching and & Engineering \\
& & & redistribution of & emergency vehicles on \\
& & & the highway network & \\
\hline
\end{tabular}


Cite as: Maaroof, B.B., Rashid, T.A., Abdulla, J.M. et al. (2022). Current Studies and Applications of Shuffled Frog Leaping Algorithm: A Review. Arch Computat Methods Eng. https://doi.org/10.1007/s11831-021-09707-2

\begin{tabular}{|c|c|c|c|c|}
\hline [66], T. Liu et al. & 2018 & HSFLA & $\begin{array}{l}\text { Raman fiber amplifier } \\
\text { design }\end{array}$ & $\begin{array}{l}\text { Optical } \\
\text { engineering }\end{array}$ \\
\hline [67], A. Kaveh et al. & 2019 & HSFLA & $\begin{array}{l}\text { Finding an optimal } \\
\text { solution region rapidly } \\
\text { in designing trusses }\end{array}$ & $\begin{array}{l}\text { Civil } \\
\text { engineering }\end{array}$ \\
\hline [68], N. R. Babu et al. & 2020 & HSFLA & $\begin{array}{l}\text { Optimizing TIDN } \\
\text { controller values }\end{array}$ & Engineering \\
\hline $\begin{array}{l}\text { [69], A. Ghaemi and B. } \\
\text { Arasteh }\end{array}$ & 2020 & MSFLA & $\begin{array}{l}\text { Generating structural } \\
\text { test data for automatic } \\
\text { test data generation in } \\
\text { software engineering }\end{array}$ & $\begin{array}{l}\text { Software } \\
\text { engineering }\end{array}$ \\
\hline [70], F. Jiang et al. & 2020 & HSFLA & $\begin{array}{l}\text { Inversion of } 2 \mathrm{D} \\
\text { electrical resistivity } \\
\text { problem }\end{array}$ & Engineering \\
\hline $\begin{array}{l}{[14], \text { D. Lei and } \mathrm{T} \text {. }} \\
\text { Wang }\end{array}$ & 2019 & HSFLA & $\begin{array}{l}\text { Investigating } \\
\text { distributed two-stage } \\
\text { hybrid flow shop } \\
\text { scheduling problem } \\
\text { (DTHFSP) }\end{array}$ & Engineering \\
\hline $\begin{array}{l}\text { [27], H.-P. Hsu and T.- } \\
\text { L. Chiang }\end{array}$ & 2019 & MSFLA & $\begin{array}{l}\text { Organizing dynamic } \\
\text { and continuous berth } \\
\text { allocation problem } \\
\text { (DCBAP) in which } \\
\text { both arrived and } \\
\text { incoming ships }\end{array}$ & $\begin{array}{l}\text { Engineering } \\
\text { management }\end{array}$ \\
\hline [64], D. Lei et al. & 2017 & SFLA & $\begin{array}{l}\text { Solving a flexible job } \\
\text { shop problem (FJSP) } \\
\text { for energy consumption }\end{array}$ & Engineering \\
\hline [71], X. Tao et al. & 2019 & MSFLA & $\begin{array}{l}\text { Power load-constrained } \\
\text { time-cost-resource } \\
\text { trade-off (TCRTO). }\end{array}$ & $\begin{array}{l}\text { Civil } \\
\text { engineering }\end{array}$ \\
\hline
\end{tabular}

\section{Operational framework of SFLA}

As mentioned in the previous sections before, SFLA and its extension have been utilized in a variety of applications. Each SFLA variation application requires a unique process, although several SFLA variations may be used to address a given kind of issue, or several issues may be addressed with the same kind of SFLA variation. As a result, we present an operational framework for SFLA variations, which serves as a concise description of their applications and methods in environments and water resources, computing, power and energy, and engineering problems. Figure (6) illustrates the 
proposed framework. The main extensions of SFLA are HSFLA and HSFLA. The standard SFLA and its enhanced versions have been used to a variety of real-world applications. The standard SFLA has been deployed on nine types of different application problems. Similarly, MHSFA has been applied on nine different real-world applications. On the other hand, HSFLA was the most used one on different practical applications. It has been exploited on ten variety of practical problems. Meanwhile, the SFLA and its extensions have been used for the same problem in some cases. For instance, all the three mentioned algorithms have been utilized for flexible job/flow shop scheduling problem and power distribution grid and controller.

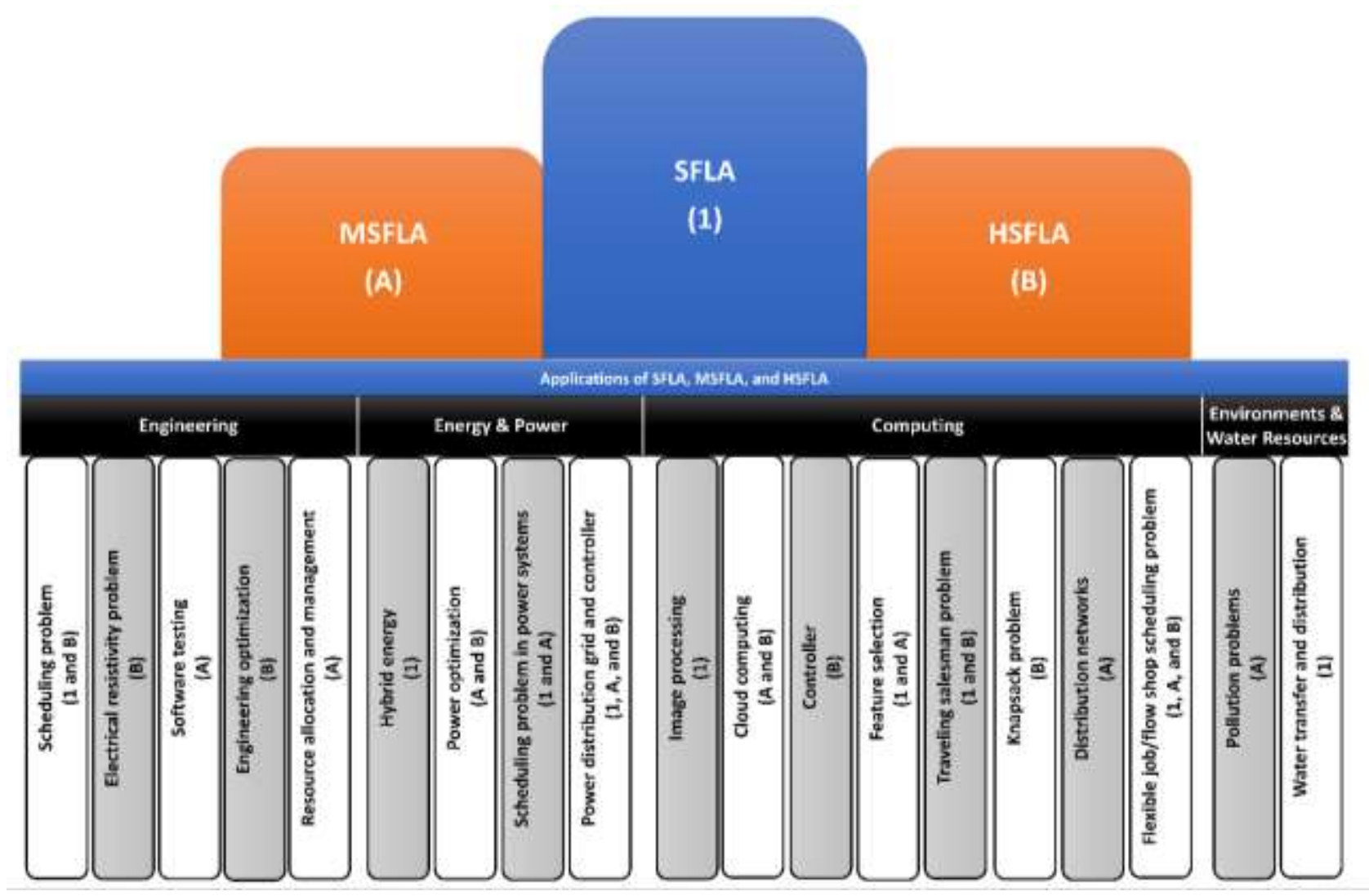

Figure (6): The proposed operational framework of SFLA

\section{Conclusion and Prospects}

In this review, a comprehensive survey was conducted, which can be used as a useful source by the scholars who desire to add an impact in the algorithm or apply it to solve real-world applications. In this work, we mentioned the most relevant and recent works applied in various areas, such as modifications done on the different parts of the SFLA. Also, the hybridization of the SFLA with other algorithms, and then, the applications of the algorithm were discussed. Finally, an operational framework for analyzing the usage of SLFA and its variations presented. 
Cite as: Maaroof, B.B., Rashid, T.A., Abdulla, J.M. et al. (2022). Current Studies and Applications of Shuffled Frog Leaping Algorithm: A Review. Arch Computat Methods Eng. https://doi.org/10.1007/s11831-021-09707-2

It can be seen from the statistics that the algorithm was in several real-world problems due to its simple implementation. Besides, it provides a solution to the complex problem as well due to its fast convergence. The performance of the algorithm was evaluated against other competitive algorithms, mostly with PSO and GA. In general, the SFLA has shown competitive results against both algorithms. From the modifications and hybridizations, which were done in the area, we can conclude that the SFLA has several aspects for researchers to work on, such as memeplex grouping and shuffling mechanism, parameter selection, virtual frog population creation, partitioning of frogs through the memeplexes, best and worst frog selection, and worst frog position updating formula. These limitations were overcome in local and global explorations for different proposes as preventing the algorithm from trapping into local optima and increasing the convergence rate.

Although SFLA and its variants were implemented to solve many complex problems, still some researchers claim some limitations in several aspects.

- Narrow local search space [21], since the worst frog can never jump over the best frog, which leads the algorithm to trap in local optima, reduces the convergence speed and precipitate convergence;

- Most of the publications claim about the slow convergence of the algorithm as well as trapping into local optima, and premature convergence [17, 20, 22, 24];

- Unbalanced memeplex performance [25];

- Long execution time [28].

- Because of its simplicity in implementation and the fewer parameters, many works have applied the SFLA on different applications and besides, the modifications, as well as hybridizations of the algorithm have been a hot spot for the scholars. But still, there is room for others to work in improving the algorithm as it can be hybridized with most recent swarm algorithms, such as backtracking search optimization algorithm [12, 13], the variants of evolutionary clustering algorithm star [72-75], chaotic sine cosine firefly algorithm [76], and hybrid artificial intelligence algorithms [77]. Furthermore, HS can be applied to more complex and real-world applications to explore more deeply the advantages and drawbacks of the algorithm or improve its efficiencies, such as engineering application problems [76], laboratory management [78], e-organization and e-government services [79], online analytical processing [80], web science [81], the Semantic Web ontology learning [82], chronic wound image processing [83], signal detection processing [84], and concept drift detection in big social data [85]. 
Cite as: Maaroof, B.B., Rashid, T.A., Abdulla, J.M. et al. (2022). Current Studies and Applications of Shuffled Frog Leaping Algorithm: A Review. Arch Computat Methods Eng. https://doi.org/10.1007/s11831-021-09707-2

\section{Acknowledgements}

Some special thanks go to Kurdistan Institution for Strategic Studies and Scientific Research and the University of Kurdistan Hewler for its help and willingness to conduct this review.

\section{Compliance with Ethical Standards}

\section{Conflict of interest: None.}

Funding: No funding is applicable.

\section{References}

1. Duan Q, Sorooshian S, Gupta V (1992) Effective and efficient global optimization for conceptual rainfall-runoff models. Water Resour Res 28:1015-1031

2. Kennedy J, Eberhart R (1995) Particle swarm optimization. In: Proceedings of ICNN'95international conference on neural networks. IEEE, pp 1942-1948

3. Eusuff M, Lansey K, Pasha F (2006) Shuffled frog-leaping algorithm: a memetic metaheuristic for discrete optimization. Eng Optim 38:129-154

4. Eusuff MM, Lansey KE (2003) Optimization of water distribution network design using the shuffled frog leaping algorithm. J Water Resour Plan Manag 129:210-225

5. Liong S-Y, Atiquzzaman M (2004) Optimal design of water distribution network using shuffled complex evolution. J Inst Eng Singapore 44:93-107

6. Lei J (2011) Generalized reconstruction algorithm for compressed sensing. Comput Electr Eng 37:570-588

7. Sarkheyli A, Zain AM, Sharif S (2015) The role of basic, modified and hybrid shuffled frog leaping algorithm on optimization problems: a review. Soft Comput 19:2011-2038

8. Luo X, Yang Y, Li X (2008) Solving TSP with shuffled frog-leaping algorithm. In: 2008 Eighth International Conference on Intelligent Systems Design and Applications. IEEE, pp $228-232$

9. Li W, Pang J, Niu Q, Zhang W (2015) Application of Improved Support Vector Machine Based on Shuffled Frog Leaping Algorithm in Wind-Photovoltaic-Battery Power Forecasting. In: 2015 7th International Conference on Intelligent Human-Machine Systems and Cybernetics. IEEE, pp 128-131

10. Mohammed HM, Umar SU, Rashid TA (2019) A Systematic and Meta-Analysis Survey of Whale Optimization Algorithm. Comput Intell Neurosci 2019:

11. Rahman CM, Rashid TA (2019) Dragonfly algorithm and its applications in applied science survey. Comput Intell Neurosci 2019:

12. Hassan BA, Rashid TA (2019) Operational framework for recent advances in backtracking search optimisation algorithm: A systematic review and performance evaluation. Appl Math 
Cite as: Maaroof, B.B., Rashid, T.A., Abdulla, J.M. et al. (2022). Current Studies and Applications of Shuffled Frog Leaping Algorithm: A Review. Arch Computat Methods Eng. https://doi.org/10.1007/s11831-021-09707-2

\section{Comput 124919}

13. Hassan BA, Rashid TA (2020) Datasets on statistical analysis and performance evaluation of backtracking search optimisation algorithm compared with its counterpart algorithms. Data Br 28:105046

14. Lei D, Wang T (2020) Solving distributed two-stage hybrid flowshop scheduling using a shuffled frog-leaping algorithm with memeplex grouping. Eng Optim 52:1461-1474

15. Huang Y, Shen X-N, You X (2021) A discrete shuffled frog-leaping algorithm based on heuristic information for traveling salesman problem. Appl Soft Comput 102:107085

16. Elattar EE (2019) Environmental economic dispatch with heat optimization in the presence of renewable energy based on modified shuffle frog leaping algorithm. Energy 171:256-269

17. Moazzami M, Gharehpetian GB, Shahinzadeh H, Hosseinian SH (2017) Optimal locating and sizing of DG and D-STATCOM using Modified Shuffled Frog Leaping Algorithm. In: 2017 2nd Conference on Swarm Intelligence and Evolutionary Computation (CSIEC). IEEE, pp 54-59

18. Oboudi MH, Hooshmand R, Karamad A (2016) Feasible method for making controlled intentional islanding of microgrids based on the modified shuffled frog leap algorithm. Int $\mathrm{J}$ Electr Power Energy Syst 78:745-754

19. Mora-Melia D, Iglesias-Rey PL, Martínez-Solano FJ, Muñoz-Velasco P (2016) The efficiency of setting parameters in a modified shuffled frog leaping algorithm applied to optimizing water distribution networks. Water 8:182

20. Baziar A, Jabbari M, Shafiee H (2015) A new method based on modified shuffled frog leaping algorithm in order to solve nonlinear large scale problem. Int J Sci Technol Res 4:149-154

21. Huynh T-H (2008) A modified shuffled frog leaping algorithm for optimal tuning of multivariable PID controllers. In: 2008 IEEE International Conference on Industrial Technology. IEEE, pp 1-6

22. Elbeltagi E, Hegazy T, Grierson D (2007) A modified shuffled frog-leaping optimization algorithm: applications to project management. Struct Infrastruct Eng 3:53-60

23. Duan X, Song S, Zhao J (2015) Emergency vehicle dispatching and redistribution in highway network based on bilevel programming. Math Probl Eng 2015:

24. Naruka B, Sharma TK, Pant M, et al (2014) Two-phase shuffled frog-leaping algorithm. In: Proceedings of 3rd International Conference on Reliability, Infocom Technologies and Optimization. IEEE, pp 1-5

25. Zhen Z, Wang D, Liu Y (2009) Improved shuffled frog leaping algorithm for continuous optimization problem. In: 2009 IEEE Congress on Evolutionary Computation. IEEE, pp 2992-2995

26. Siahbalaee J, Rezanejad N, Gharehpetian GB (2019) Reconfiguration and DG sizing and placement using improved shuffled frog leaping algorithm. Electr Power Components Syst 47:1475-1488

27. Hsu H-P, Chiang T-L (2019) An improved shuffled frog-leaping algorithm for solving the 
dynamic and continuous berth allocation problem (DCBAP). Appl Sci 9:4682

28. Zhang T, Zhao X, Pan X, et al (2018) Optimal local dimming based on an improved shuffled frog leaping algorithm. IEEE Access 6:40472-40484

29. Hu B, Dai Y, Su Y, et al (2016) Feature selection for optimized high-dimensional biomedical data using an improved shuffled frog leaping algorithm. IEEE/ACM Trans Comput Biol Bioinforma 15:1765-1773

30. Pu H, Zhen Z, Wang D (2011) Modified shuffled frog leaping algorithm for optimization of UAV flight controller. Int J Intell Comput Cybern

31. Azizipanah-Abarghooee R, Narimani MR, Bahmani-Firouzi B, Niknam T (2014) Modified shuffled frog leaping algorithm for multi-objective optimal power flow with FACTS devices. J Intell Fuzzy Syst 26:681-692

32. Cai J, Zhou R, Lei D (2020) Dynamic shuffled frog-leaping algorithm for distributed hybrid flow shop scheduling with multiprocessor tasks. Eng Appl Artif Intell 90:103540

33. Guo Y, Tian X, Fang G, Xu Y-P (2020) Many-objective optimization with improved shuffled frog leaping algorithm for inter-basin water transfers. Adv Water Resour 138:103531

34. Yan L, Chen B, Xu J, et al Research on Optimal PMU Placement Method Considering Node Failure Rate

35. Naruka B, Sharma TK, Pant M, et al (2015) Differential shuffled frog-leaping algorithm. In: Proceedings of Fourth International Conference on Soft Computing for Problem Solving. Springer, pp 249-257

36. Lu Z, Wang C, Guo J (2018) A hybrid of fish swarm algorithm and shuffled frog leaping algorithm for attribute reduction. In: 2018 13th World Congress on Intelligent Control and Automation (WCICA). IEEE, pp 1482-1487

37. Wang L, Gong Y (2013) Quantum binary shuffled frog leaping algorithm. In: 2013 Third International Conference on Instrumentation, Measurement, Computer, Communication and Control. IEEE, pp 1655-1659

38. Li W, Cao J, Wu J, et al (2018) A collaborative filtering recommendation method based on discrete quantum-inspired shuffled frog leaping algorithms in social networks. Futur Gener Comput Syst 88:262-270

39. Tang Z, Luo K (2014) K-means Clustering Algorithm Method Based on Shuffled Frog Leaping Algorithm. In: Advanced Materials Research. Trans Tech Publ, pp 2245-2249

40. Li Y, Yan Z (2019) Improved shuffled frog leaping algorithm on system reliability analysis. Brain informatics 6:1-7

41. Nie X, Nie H (2017) MPPT control strategy of PV based on improved shuffled frog leaping algorithm under complex environments. J Control Sci Eng 2017:

42. Ibrahim GJ, Rashid TA, Akinsolu MO (2020) An energy efficient service composition mechanism using a hybrid meta-heuristic algorithm in a mobile cloud environment. J Parallel Distrib Comput 143:77-87 
43. Liu G, Chen L, Shen Z, et al (2019) A fast and robust simulation-optimization methodology for stormwater quality management. J Hydrol 576:520-527

44. Sharma TK, Prakash D (2020) Air pollution emissions control using shuffled frog leaping algorithm. Int J Syst Assur Eng Manag 11:332-339

45. Prakash D, Tripathi A, Sharma TK (2018) Application of shuffled frog-leaping algorithm in regional air pollution control. In: Soft Computing: Theories and Applications. Springer, pp $397-403$

46. Boroumand B, Yaghoubi E, Barekatain B (2021) An enhanced cost-aware mapping algorithm based on improved shuffled frog leaping in network on chips. J Supercomput 77:498-522

47. Mohammadhosseini M, Najafzadeh S, Mahdipour E (2021) Reduce energy consumption in sensors using a smartphone, smartwatch, and the use of SFLA algorithms (REC-SSS). J Supercomput 77:909-935

48. Khadanga RK, Kumar A, Panda S (2020) A hybrid shuffled frog-leaping and pattern search algorithm for load frequency controller design of a two-area system composing of PV grid and thermal generator. Int J Numer Model Electron Networks, Devices Fields 33:e2694

49. Karpagam M, Geetha K, Rajan C (2020) A modified shuffled frog leaping algorithm for scientific workflow scheduling using clustering techniques. Soft Comput 24:637-646

50. Kayalvili S, Selvam M (2019) Hybrid SFLA-GA algorithm for an optimal resource allocation in cloud. Cluster Comput 22:3165-3173

51. Lei D, Guo X (2016) A shuffled frog-leaping algorithm for job shop scheduling with outsourcing options. Int J Prod Res 54:4793-4804

52. Karakoyun M (2019) A New Approach Based on K-means Clustering and Shuffled Frog Leaping Algorithm to Solve Travelling Salesman Problem. Acad Perspect Procedia 2:446453

53. Wang L, Guo C, Li Y, et al (2019) An outsourcing service selection method using ANN and SFLA algorithms for cement equipment manufacturing enterprises in cloud manufacturing. $\mathrm{J}$ Ambient Intell Humaniz Comput 10:1065-1079

54. Pérez-Delgado M-L (2019) Color image quantization using the shuffled-frog leaping algorithm. Eng Appl Artif Intell 79:142-158

55. Yuvaraj N, Sabari A (2017) Twitter sentiment classification using binary shuffled frog algorithm. Intell Autom Soft Comput 23:373-381

56. Amudha A (2014) Optimal placement of Unified Power Flow Controller in the transmission line using SLF algorithm. Appl Mech Mater

57. Mosayebian ME, Soleymani S, Mozafari B, Shayanfar HA (2016) Optimal operational management of a micro-grid, including high penetration level of renewable energy resources. J Intell Fuzzy Syst 31:565-577

58. Gitizadeh M, Vahed AA, Aghaei J (2013) Multistage distribution system expansion planning considering distributed generation using hybrid evolutionary algorithms. Appl Energy 101:655-666 
59. Reddy AS, Vaisakh K (2013) Shuffled differential evolution for large scale economic dispatch. Electr Power Syst Res 96:237-245

60. Sridhar R, Jeevananthan S, Dash SS, Vishnuram P (2017) A new maximum power tracking in PV system during partially shaded conditions based on shuffled frog leap algorithm. J Exp Theor Artif Intell 29:481-493

61. Nagaraju S, Reddy AS, Vaisakh K (2019) Shuffled differential evolution-based combined heat and power economic dispatch. In: Soft Computing in Data Analytics. Springer, pp 525532

62. Hu P, Pan J-S, Chu S-C, et al (2019) New hybrid algorithms for prediction of daily load of power network. Appl Sci 9:4514

63. Chamandoust H, Derakhshan G, Bahramara S (2020) Multi-objective performance of smart hybrid energy system with Multi-optimal participation of customers in day-ahead energy market. Energy Build 216:109964

64. Lei D, Zheng Y, Guo X (2017) A shuffled frog-leaping algorithm for flexible job shop scheduling with the consideration of energy consumption. Int J Prod Res 55:3126-3140

65. Siadatan A, Taheri B, Sedaghat M (2019) New fundamental modulation technique with SHE using shuffled frog leaping algorithm for multilevel inverters. Evol Syst 1-17

66. Liu T, Liu L, Chen J, et al (2018) Optimal design of Raman fibre amplifier based on terminal value optimization strategy and shuffled frog leaping algorithm. J Mod Opt 65:1680-1687

67. Kaveh A, Talatahari S, Khodadadi N (2019) Hybrid invasive weed optimization-shuffled frog-leaping algorithm for optimal design of truss structures. Iran J Sci Technol Trans Civ Eng 1-16

68. Babu NR, Saikia LC, Raju DK, Chiranjeevi T (2020) Maiden Application of Hybrid Shuffled Frog-Leaping Algorithm with Pattern Search Algorithm in AGC Studies of a Multiarea System. In: Computing Algorithms with Applications in Engineering. Springer, pp 335348

69. Ghaemi A, Arasteh B (2020) SFLA-based heuristic method to generate software structural test data. J Softw Evol Process 32:e2228

70. Jiang F, Dong L, Dai Q (2020) Electrical Resistivity Inversion Based on a Hybrid CCSFLAMSVR Method. Neural Process Lett 51:2871-2890

71. Tao X, Li H, Mao C, et al (2019) Developing shuffled frog-leaping algorithm (SFLA) method to solve power load-constrained TCRTO problems in civil engineering. Adv Civ Eng 2019:

72. Hassan BA, Rashid TA (2021) A multidisciplinary ensemble algorithm for clustering heterogeneous datasets. Neural Comput Appl. https://doi.org/10.1007/s00521-020-05649-1

73. Hassan BA, Rashid TA, Hamarashid HK (2021) A Novel Cluster Detection of COVID-19 Patients and Medical Disease Conditions Using Improved Evolutionary Clustering Algorithm Star. Comput Biol Med 104866

74. Hassan BA, Rashid TA, Mirjalili S (2021) Formal context reduction in deriving concept hierarchies from corpora using adaptive evolutionary clustering algorithm star. Complex 
Cite as: Maaroof, B.B., Rashid, T.A., Abdulla, J.M. et al. (2022). Current Studies and Applications of Shuffled Frog Leaping Algorithm: A Review. Arch Computat Methods Eng. https://doi.org/10.1007/s11831-021-09707-2

Intell Syst 1-16

75. Hassan BA, Rashid TA, Mirjalili S (2021) Performance evaluation results of evolutionary clustering algorithm star for clustering heterogeneous datasets. Data Br 107044

76. Hassan BA (2020) CSCF: a chaotic sine cosine firefly algorithm for practical application problems. Neural Comput Appl 1-20

77. Hassan BA, Rashid TA (2021) Artificial Intelligence Algorithms for Natural Language Processing and the Semantic Web Ontology Learning. arXiv Prepr arXiv210813772

78. Saeed MHR, Hassan BA, Qader SM (2017) An Optimized Framework to Adopt Computer Laboratory Administrations for Operating System and Application Installations. Kurdistan J Appl Res 2:92-97

79. Hassan BA, Ahmed AM, Saeed SA, Saeed AA (2016) Evaluating e-Government Services in Kurdistan Institution for Strategic Studies and Scientific Research Using the EGOVSAT Model. Kurdistan J Appl Res 1:1-7

80. Hassan BA, Qader SM A New Framework to Adopt Multidimensional Databases for Organizational Information Sys-tem Strategies

81. Hassan BA (2021) Analysis for the overwhelming success of the web compared to microcosm and hyper-G systems. arXiv Prepr arXiv210508057

82. Hassan B, Dasmahapatra S Towards Semantic Web: Challenges and Needs

83. Chakraborty C (2017) Chronic wound image analysis by particle swarm optimization technique for tele-wound network. Wirel Pers Commun 96:3655-3671

84. Munirathinam R, Ponnan S, Chakraborty C, Umathurai S (2021) Improved performance on seizure detection in an automated electroencephalogram signal under evolution by extracting entropy feature. Multimed Tools Appl 1-16

85. Abbasi A, Javed AR, Chakraborty C, et al (2021) ElStream: An Ensemble Learning Approach for Concept Drift Detection in Dynamic Social Big Data Stream Learning. IEEE Access 9:66408-66419 